\title{
Model-Based Clustering and New Edge Modelling in Large Computer Networks
}

\author{
Silvia Metelli \\ Imperial College London \\ s.metelli13@imperial.ac.uk
}

\author{
Nicholas Heard \\ Imperial College London \& \\ Heilbronn Institute, University of Bristol \\ n.heard@imperial.ac.uk
}

\begin{abstract}
Computer networks are complex and the analysis of their structure in search for anomalous behaviour is both a challenging and important task for cyber security. For instance, new edges, i.e. connections from a host or user to a computer that has not been connected to before, provide potentially strong statistical evidence for detecting anomalies. Unusual new edges can sometimes be indicative of both legitimate activity, such as automated update requests permitted by the client, and illegitimate activity, such as denial of service (DoS) attacks to cause service disruption or intruders escalating privileges by traversing through the host network. In both cases, capturing and accumulating evidence of anomalous new edge formation represents an important security application. Computer networks tend to exhibit an underlying cluster structure, where nodes are naturally grouped together based on similar connection patterns. What constitutes anomalous behaviour may strongly differ between clusters, so inferring these peer groups constitutes an important step in modelling the types of new connections a user would make.
\end{abstract}

In this article, we present a two-step Bayesian statistical method aimed at clustering similar users inside the network and simultaneously modelling new edge activity, exploiting both overall-level and cluster-level covariates.

\section{INTRODUCTION}

The widespread growth of cyber attacks and the imperative need to keep computer networks secure has led analysts to develop new intrusion detection systems to respond to these sophisticated attacks. In particular, statistical anomaly detection has recently become an important area of research in cyber security. This approach has been attracting attention during the last decade, due to the proliferation of traffic data together with the need to quickly analyse data in real time. However, several challenges still hinder the deployment of anomaly-based methods for large computer networks such as computational speed and scalability. Anomaly-based methods screen the network looking for abnormal deviations from a statistical model of the normal state of the system [1]. No prior knowledge about the characteristics of an attack is needed, allowing zero-day attacks to be identified. This is in contrast to popular signature-based intrusion detection systems, which can only detect attacks for which a signature has previously been created [2]. In this paper, we take an anomaly-based approach, aiming at modelling the arrivals of new edges within the large enterprise computer network of Los Alamos National Laboratory (LANL). While previous work on new edge modelling has focused on the rate of occurrence of new edges [3], here we investigate the identity of the new edges. Specifically, we need a reliable and scalable model to monitor new connections occurring in the network, where the characteristics of each new edge are analysed on the basis of past behaviour of each network host. In this context, identifying groups of hosts sharing similar connections is fundamental to indicate if shared connectivity can be predictive of similar future interactions. Computer networks are typically large and decomposing the network graph looking for peer groups can reveal hidden network structures and provide useful information about the likely identity of new connections, aiding the problem of new edge prediction.

Towards this end, we present a method based on a sequential two-step Bayesian inference procedure to simultaneously infer cluster configurations and model new edge formation. A Bayesian agglomerative model-based clustering algorithm is used as a first step of the analysis, with the purpose of identifying an initial, reliable cluster configuration of users sharing similar connection behaviour. Subsequently, the identity of new edges is modelled through a Bayesian Cox proportional hazards model, where the initial cluster configuration and the model coefficient parameters are jointly updated with a Markov Chain Monte Carlo (MCMC) simulation, in order to improve the predictive performance of the initial cluster configurations. The procedure is repeated sequentially through a linear updating scheme, as new data arrive.

The remainder of the paper is structured as follows. In Section II we present a model for new edge formation. A description of Bayesian model-based agglomerative clustering is provided in Section III, while Section IV introduces our procedure for performing posterior inference. Section V describes the data set used for the application and presents the main results of the analysis. Section VI concludes.

\section{A BAYESIAN COX PROPORTIONAL HAZARDS MODEL FOR NEW EDGES}

The computer network is here encoded as a graph of authenticated connections between a set of users $\mathcal{U}$ and a set of computers $\mathcal{C}$, which can be naturally represented as a bipartite graph. Users and computers represent two 
sets of disjoint nodes of the computer network and the presence of connections between them form the edges. From the start of an observation period of the computer network, new connections from users to computers are observed, each adding a new edge to the bipartite graph. Let $\mathcal{D}=\left\{\left(u_{1}, c_{1}\right), \ldots,\left(u_{n}, c_{n}\right)\right\}$ be the time-ordered sequence of new (user, computer) edges observed in the network graph. We model new edge formation in the graph as a Cox proportional hazards model [4] with time-dependent covariates. This model conveniently integrates the benefits of non-parametric and parametric approaches to statistical inferences by dividing the hazard into a non-parametric part, namely the baseline hazard and a parametric part, namely the covariate effects.

In this analysis, the hazard of observing a new connection between a particular user $i$ and computer $j$ at time $t$ is modelled as the product of a baseline hazard and the exponential of a linear combination of the covariates. One relevant time-varying covariate is the degree of the computer, which measures the number of unique authentications over time, thus providing a sort of 'popularity' effect of each computer. We include two different degree effects: one representing the degree of computer $j$ measured on the overall network graph at time $t$, denoted $N_{j}(t)$, and one representing the degree of the computer amongst a group $B$ of users considered to be similar to the user $i$ in question, denoted $N_{B, j}$. This allows us to account for cluster-specific effects, which as previously mentioned, could be fundamental to improving the predictive ability of the model. As detailed in the next section, clusters are here defined as subsets of $\mathcal{U}$ and a cluster configuration $\mathfrak{C}=\left\{\mathfrak{C}_{1}, \ldots, \mathfrak{C}_{K}\right\}$ is a partition of $\mathcal{U}$ into such subsets. For user $i$, let $k(i) \in \mathbb{C}$ be the corresponding user cluster. The overall and cluster-specific covariates for computer $j$ are respectively defined as

$$
\begin{aligned}
& N_{j}(t)=\sum_{t^{\prime}=1}^{t-1} \mathbb{1}\left(c_{t^{\prime}}=j\right), \\
& N_{B, j}(t)=\sum_{t^{\prime}=1}^{t-1} \mathbb{1}\left(c_{t^{\prime}}=j\right) \mathbb{1}\left(u_{t^{\prime}} \in B\right), B \subseteq \mathcal{U} .
\end{aligned}
$$

Note that $N_{j}(t) \equiv N_{\mathcal{U}, j}(t)$. Let $\bar{\lambda}_{i}(t)$ be a baseline hazard for new edge formation for user $i$ and

$$
\mathcal{C}_{t}(i)=\left\{j \in \mathcal{C} \mid \exists t^{\prime}<t \text { s.t. } u_{t^{\prime}}=i, c_{t^{\prime}}=j\right\}
$$

be the subset of $\mathcal{C}$ containing the computers to which user $i$ authenticated to by time $t$. For a user $i$ and computer $j$, the hazard function takes the following form

$$
\lambda_{i j}(t)=\bar{\lambda}_{i}(t) \exp \left\{\alpha_{1} N_{j}(t)+\alpha_{2, k(i)} N_{k(i), j}(t)\right\} \mathbb{1}\left(j \notin \mathcal{C}_{t}(i)\right) .
$$

Cox proportional hazards models consider the baseline rate $\bar{\lambda}_{i}(t)$ as a nuisance parameter and so the coefficient parameter vector can be estimated using a so-called partial likelihood. For the sequence $\mathcal{D}$ of new edges, the partial likelihood at time $t$ is given by

$$
\begin{aligned}
P L(\mathcal{D} \mid \boldsymbol{\alpha}, \mathfrak{C}) & =\prod_{t=1}^{n} \frac{\lambda_{u_{t} c_{t}}(t)}{\sum_{j \in \mathcal{C}} \lambda_{u_{t} j}(t)}= \\
& =\prod_{t=1}^{n} \frac{\exp \left\{\alpha_{1} N_{c_{t}}(t)+\alpha_{2, k\left(u_{t}\right)} N_{k\left(u_{t}\right), c_{t}}(t)\right\}}{\sum_{j \notin \mathcal{C}_{t}\left(u_{t}\right)} \exp \left\{\alpha_{1} N_{j}(t)+\alpha_{2, k\left(u_{t}\right)} N_{k\left(u_{t}\right), j}(t)\right\}},
\end{aligned}
$$

where $\alpha_{1} \in \mathbb{R}, \alpha_{2}=\left(\alpha_{2,1}, \ldots, \alpha_{2, K}\right) \in \mathbb{R}^{K}, \boldsymbol{\alpha}=\left(\alpha_{1}, \alpha_{2}\right)$ and $K$ is the total number of clusters in $\mathfrak{C}$. This partial likelihood allows for estimation of covariates of the model without any restrictions placed on the baseline hazard.

A Bayesian formulation of the model requires the specification of prior distributions, in order to obtain the joint posterior distribution $P(\boldsymbol{\alpha}, \mathfrak{C} \mid \mathcal{D})$ of the Cox model parameters and the cluster configuration. We choose standard normal distributions for the parameters $\boldsymbol{\alpha}$ and we assume exchangeability when specifying a prior for the cluster configuration $\mathfrak{C}$ so that a priori no two observations are more likely to belong to the same cluster. Specifically, we use a uniform distribution over the space of all possible cluster configurations. Alternatively, a Dirichlet process prior could be employed [5]. Given the model likelihood provided in Eq. (2) and prior distributions $P(\boldsymbol{\alpha} \mid \mathfrak{C})$ and $P(\mathfrak{C})$ for $\boldsymbol{\alpha}$ and $\mathfrak{C}$, the joint posterior distribution is then given by

$$
P(\boldsymbol{\alpha}, \mathfrak{C} \mid \mathcal{D}) \propto P L(\mathcal{D} \mid \boldsymbol{\alpha}, \mathfrak{C}) P(\boldsymbol{\alpha} \mid \mathfrak{C}) P(\mathfrak{C}) .
$$

Exact inference is intractable, so Markov Chain Monte Carlo (MCMC) is required to perform posterior inference. The MCMC sampling scheme used for this analysis is introduced in Section IV, while the technique used to infer some initial clusters is presented in the next section. This technique will use a simpler, surrogate model that nonetheless provides an initial cluster configuration for Eq. (3) which can then be further updated according to the Cox model, as described in Section IV.

\section{Model-Based Agglomerative Clustering}

Clustering is the task of grouping similar objects. Many different approaches have been developed in the literature, including the common hierarchical clustering [6] and modelbased clustering [7] methods. We focus here on Bayesian model-based agglomerative clustering. Agglomerative clustering is an iterative strategy which outputs a hierarchy of cluster configurations. Each data point is at first assigned to its own cluster and successively the cluster pairs which maximise an objective function are merged. A model-based approach defines a probability model as the clustering objective function, 
whilst the Bayesian formulation enables the partition of items into subsets to be a parameter of the probability model, subject to prior assumptions. A surrogate clustering model is presented below. This is used to seed the main inference procedure with the Cox proportional hazards model.

\section{A. Surrogate clustering model}

Let $X=\left(x_{i j}\right)$ be a $|\mathcal{U}| \times|\mathcal{C}|$ binary matrix representing edges in the bipartite graph between users and computers, where $x_{i j}=1$ if and only if $(i, j) \in \mathcal{D}$, i.e. user $i$ connected to computer $j$ at least once. Here, we focus on clustering the users, with the purpose of dividing the data matrix $X$ into $K$ row blocks (after a permutation). For the user set $\mathcal{U}$, the cluster configuration $\mathbb{C}$ is a partition of the index set of users $\{1, \ldots,|\mathcal{U}|\}$ into $K$ non-empty subsets $\left\{\mathfrak{C}_{1}, \ldots, \mathfrak{C}_{K}\right\}$, where the $i^{t h}$ user is allocated to the $k^{t h}$ cluster if and only if $i \in \mathbb{C}_{k}$. Let $\boldsymbol{\theta}$ be a $K \times|\mathcal{C}|$ matrix of cluster-specific parameters, such that $\theta_{k j}$ is the probability that a user in cluster $k$ will connect to computer $j$. For the data matrix $X$, the implied likelihood function for the cluster configuration and parameter is given by

$$
L(X \mid \mathfrak{C}, \boldsymbol{\theta})=\prod_{k=1}^{K} \prod_{i \in \mathfrak{C}_{k}} \prod_{j=1}^{|\mathcal{C}|} \theta_{k j}^{x_{i j}}\left(1-\theta_{k j}\right)^{1-x_{i j}} .
$$

Again, the specification of prior distributions is needed for obtaining the joint posterior distribution $P(\boldsymbol{\theta}, \mathfrak{C} \mid X)$ of model parameters. We choose independent, conjugate $\operatorname{Beta}(a, b)$ priors for the probabilities $\theta_{k j}$ with density function:

$$
f\left(\theta_{k j}\right)=\frac{\Gamma(a+b)}{\Gamma(a) \Gamma(b)} \theta_{k j}^{a-1}\left(1-\theta_{k j}\right)^{b-1},
$$

and a uniform distribution over the space of all possible cluster configurations, as in Section II.

The primary interest here is the allocation of the objects to clusters and so the marginal posterior distribution of $\mathfrak{C}$ is the objective function that we seek to maximise. This can be obtained in closed-form as

$$
\begin{aligned}
& P(\mathfrak{C} \mid X) \propto P(X \mid \mathfrak{C})=\int L(X \mid \mathfrak{C}, \boldsymbol{\theta}) f(\boldsymbol{\theta}) d \boldsymbol{\theta}= \\
& =\prod_{k=1}^{K} \prod_{j=1}^{|\mathcal{C}|} \frac{\Gamma(a+b) \Gamma\left(a+m_{k j}\right) \Gamma\left(b+n_{k}-m_{k j}\right)}{\Gamma(a) \Gamma(b) \Gamma\left(a+b+n_{k}\right)},
\end{aligned}
$$

where $m_{k j}=\sum_{i \in \mathfrak{C}_{k}} x_{i j}$ and $n_{k}=\left|\mathfrak{C}_{k}\right|$ is the number of users in cluster $k$.

\section{B. Agglomerative Clustering}

Agglomerative clustering requires a measure of similarity between any pair of clusters to be specified. An intuitive choice is the multiplicative change in the posterior probability in Eq. (6) which results from merging the clusters pair:

$$
S_{k m}=\frac{P\left(\mathbb{C}^{k m} \mid X\right)}{P(\mathfrak{C} \mid X)},
$$

where $\mathbb{C}^{k m}$ represents the cluster configuration obtained from $\mathfrak{C}$ by merging cluster $k$ and cluster $m$. By Eq. (6), this quantity is equivalent to the ratio of the marginal likelihoods of the two cluster configurations, with the model parameters integrated out, enabling a fair comparison between models with a different number of parameters. The Bayesian framework automatically embodies the principle of parsimony for scientific explanation, known as Occam's razor [8], which states that simpler models will always be preferred to unnecessarily complex ones.

The algorithm is initiated with $\mathbb{C}=\{\{1\}, \ldots,\{|\mathcal{U}|\}\}$, i.e. each user is placed in its own cluster. The pair of clusters which maximise the similarity measure in Eq. (7) are then iteratively merged until all users reside in a single cluster. A nested sequence of cluster configurations is created and the optimal configuration corresponds to the configuration in the hierarchy with the largest marginal posterior probability in Eq. (6).

\section{A TWO-STEP BAYESIAN INFERENCE PROCEDURE}

The main inference procedure for the Cox model posterior distribution in Eq. (3) consists of two steps, embedded in a sequential updating scheme for computational tractability. The entire data set is divided into segments of equal length and the following procedure is repeated sequentially as new data arrives. Firstly, we perform agglomerative clustering (AC) as described in Section III, and then secondly we use Markov Chain Monte Carlo (MCMC) sampling to simultaneously update the cluster configuration $\mathfrak{C}$ and the $\boldsymbol{\alpha}$ parameters of the Cox proportional hazards model presented in Section II.

An initial cluster configuration of the first segment of data is obtained via AC; subsequently, this configuration is updated via MCMC, jointly with Cox model parameters. The new cluster configuration is passed back to the AC step and a new data segment is added, where each data point is processed sequentially as described below; this results in an extended configuration which is then updated through MCMC. The procedure is repeated until the all data have been processed.

The main motivation behind the use of a two-step scheme lies in providing a good starting cluster configuration for the MCMC sampler. Given some initial values, MCMC methods iteratively sample from a target distribution by constructing a so-called Markov chain that has the desired distribution as its stationary distribution. Although the simultaneous sampling of Cox model parameters and cluster configurations is fundamental for improving clusters predictive performance, MCMC algorithms can strongly depend on the initial values assigned to the chain. The agglomerative clustering step is therefore introduced for providing a reliable initial cluster configuration, in order to achieve subsequent MCMC convergence quickly. This proves to be necessary because of the size of the clustering problem considered, where assigning random initial configurations could irreparably affect the convergence of the chain. The two different inference steps are described below. 


\section{A. Agglomerative Clustering step}

Agglomerative clustering under the surrogate model described in section III is used to infer an initial cluster configuration. Although this algorithm generally provides a fast and efficient deterministic procedure for small data sets, large computer networks carry a higher computational burden. Here, we achieve computational saving by performing agglomerative clustering on a small initial segment of the entire data set, and then sequentially processing the remaining data. Each subsequent data point is added one at a time, initially as a new singleton cluster $k^{\prime}$ and then a possible merger with each previously created cluster $k$ is evaluated on the basis of the similarity measure $S_{k k^{\prime}}$ in Eq. (7). In this way, re-evaluation of all pairwise similarities is not required, providing a significant improvement in terms of computational speed.

\section{B. MCMC Sampling step}

The well-known Metropolis-Hastings (M-H) algorithm [9] is used to draw approximate samples from the joint posterior distribution in Eq. (3) of the Cox model parameters $\boldsymbol{\alpha}$ and the cluster configuration $\mathbb{C}$. This algorithm generates candidate parameter values from a proposal distribution which conditions on the current value of the parameter. The scheme used to sample Cox model parameters and cluster configurations is briefly presented below.

Let $\boldsymbol{\alpha}^{t}, \mathfrak{C}^{t}$ be the values of the parameter vector and cluster configuration, after $t$ iterations. At stage $t+1$ of the algorithm, with probability 0.5 a new value $\alpha_{i}^{*}$ is proposed for a uniformly sampled component $i$, conditional on the current value $\alpha_{i}^{t}$, from a normal distribution $N\left(\alpha_{i}^{t}, \sigma^{2}\right)$, with scaling parameter $\sigma$. This just perturbs the current value by some random noise. Let $\boldsymbol{\alpha}^{*}$ be equal to $\alpha_{i}^{*}$ at position $i$, and equal to $\boldsymbol{\alpha}^{t}$ everywhere else. By symmetry of the normal proposal density, the proposed vector $\boldsymbol{\alpha}^{*}$ is accepted with probability

$$
\min \left(1, \frac{P\left(\boldsymbol{\alpha}^{*}, \mathbb{C}^{t} \mid \mathcal{D}\right)}{P\left(\boldsymbol{\alpha}^{t}, \mathbb{C}^{t} \mid \mathcal{D}\right)}\right)
$$

Similarly, with probability 0.5 we randomly choose a user $u \in \mathcal{U}$ with current cluster label $k^{t}(u)$, and propose a new cluster label $k^{*}(u)$ from a discrete uniform proposal distribution over the integer set $\left\{1, \ldots, K^{t}+1\right\} /\left\{k^{t}(u)\right\}$, where $K^{t}$ is the current number of clusters in $\mathbb{C}^{t}$. The proposed value $k^{*}(u)$ suggests a new cluster configuration $\mathbb{C}^{*}$ with $K^{*}$ clusters. If $\left|\mathfrak{C}_{k^{t}(u)}\right|=1$ and $k^{*}(u) \neq K^{t}+1$, then $K^{*}=K^{t}-1$; or else if $\left|\mathfrak{C}_{k^{t}(u)}\right|>1$ and $k^{*}(u)=K^{t}+1$, then $K^{*}=K^{t}+1$. In both of these cases, the dimension of the parameter $\boldsymbol{\alpha}^{t}$ must change. Initially we set $\boldsymbol{\alpha}^{t}=\boldsymbol{\alpha}^{*}$, but if $K^{*}=K^{t}-1$ then the $k^{t}(u)$ component $\alpha_{k^{t}(u)}^{*}$ is deleted, and if $K^{*}=K^{t}+1$ then a new component $\alpha_{K^{*}}^{*}$ is proposed from the standard normal prior. Then taking, for example, the most common case where $K^{*}=K^{t}$, the resulting cluster configuration and parameter vector are accepted with probability

$$
\min \left(1, \frac{P\left(\boldsymbol{\alpha}^{t}, \mathfrak{C}^{*} \mid \mathcal{D}\right) K^{t}}{P\left(\boldsymbol{\alpha}^{t}, \mathbb{C}^{t} \mid \mathcal{D}\right) K^{*}}\right)
$$

\section{AN APPLiCATION to LOS Alamos National LABORATORY AUTHENTICATION DATA}

\section{A. Network Authentication Data}

The motivating data set consists of comprehensive authentication data [10] from the enterprise computer network at Los Alamos National Laboratory (LANL), representing 9 months of contiguous authentication activity. More specifically, the data set contains 708,304,516 time-ordered, successful user to computer authentication events among 11,362 users and 22,284 computers in the network. The timing of each authentication pair of anonymised user and anonymised computer is recorded at one second resolution.

\section{B. Results}

The proposed approach was applied to the first 10,000 distinct LANL network authentication events, with segments of 1,000 unique authentication events added at each sequential updating step. Table I provides a summary of some basic graph statistical quantities of interest for the subset of data analysed, while Figure V-B shows a log-log plot of the degree distribution of the computers over those 10,000 events. We observe that the degree distribution appears to follow a power law. In particular, the majority of the computers have only one authenticated user and only a few computers have high degree.

TABLE I

SUMMARY STATISTICS FOR THE SUBSET OF DATA ANALYSED

\begin{tabular}{|l|l|}
\hline Events & 10,000 \\
Users & 1,272 \\
Computers & 1,432 \\
Min computer degree & 1 \\
Mean computer degree & 1.86 \\
Max computer degree & 93 \\
\hline
\end{tabular}

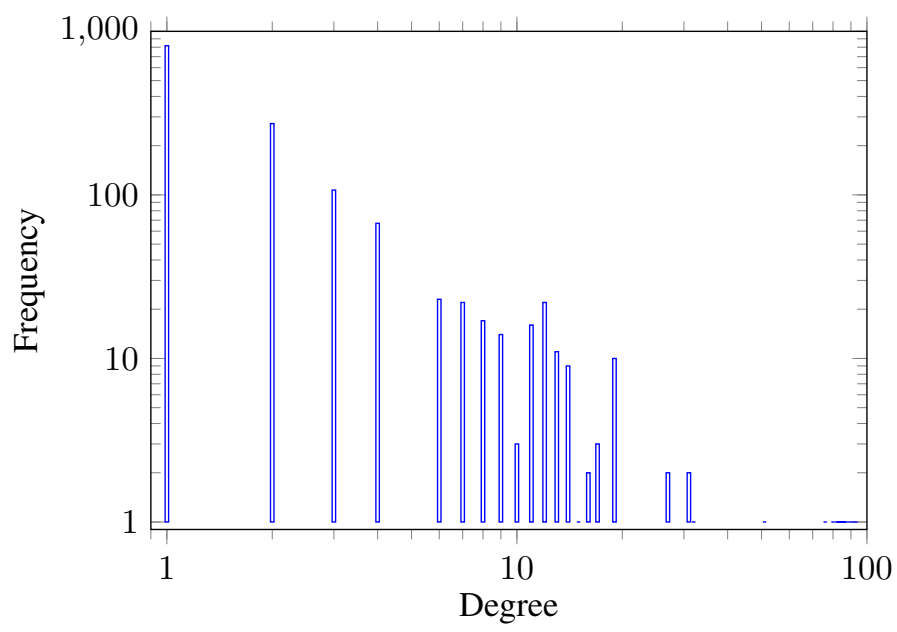

Fig. 1. Probability distribution of computer degrees (log-log scale). 
For agglomerative clustering, informative beta prior distributions were used for the probability parameter $\boldsymbol{\theta}$ : in particular, an empirical beta distribution was built for each computer, with mean equal to the proportion of users authenticated on that computer. The rationale for using informative prior distributions is the following. Computer networks show highly skewed connectivity behaviour (as in Figure V-B), where most of the nodes have few connections while some nodes are highly connected. For instance, the full LANL computer network data contain three computers with over 9,000 unique user authentications. Therefore, observing user authentications on such high-degree computers may not be informative about the user profile. Particularly for very high degree or very low degree nodes, a flat beta prior distribution would not be suitable. For MCMC, the Metropolis-Hastings algorithm was used with a total number of iterations set to 5,000, after a burn-in period of size 1,000. As previously mentioned, the prior distribution for the cluster configuration is chosen to be uniform while standard normal distributions were used for Cox model parameters.

Six clusters were identified as the most probable number and conditional on this number of clusters, Table II shows posterior means and standard deviations for the coefficients of the covariates, i.e. an overall degree effect $\alpha_{1}$ and clusterspecific degree effects $\alpha_{2}$. The estimated coefficients are all positive, indicating that a high computer degree strengthens the probability of a new user establishing a connection to that computer, particularly when users with similar connectivity patterns have also connected to that computer. Furthermore, the effect is stronger for $\alpha_{2,1}, \alpha_{2,2}$ and $\alpha_{2,3}$, which correspond to the largest clusters.

TABLE II

POSTERIOR MODEL COEFFICIENT ESTIMATES AND STANDARD ERRORS

\begin{tabular}{c|c|c}
\hline Coefficient & Mean & Standard Error \\
\hline \hline$\alpha_{1}$ & 1.06 & 0.0052 \\
$\alpha_{2,1}$ & 2.25 & 0.0737 \\
$\alpha_{2,2}$ & 2.01 & 0.0312 \\
$\alpha_{2,3}$ & 1.97 & 0.0571 \\
$\alpha_{2,4}$ & 0.96 & 0.0331 \\
$\alpha_{2,5}$ & 1.11 & 0.0211 \\
$\alpha_{2,6}$ & 0.43 & 0.0337
\end{tabular}

The heat map in Figure 2 shows the clustered data matrix, where the greyscale indicates different cluster allocations. Within the six user clusters identified, there are two dominant groups corresponding to the clusters accounting for the highest posterior coefficient effects.

Furthermore, the appropriateness of the probability model used is investigated through an analysis of the model's predictive performance. Assessing the plausibility of a posited

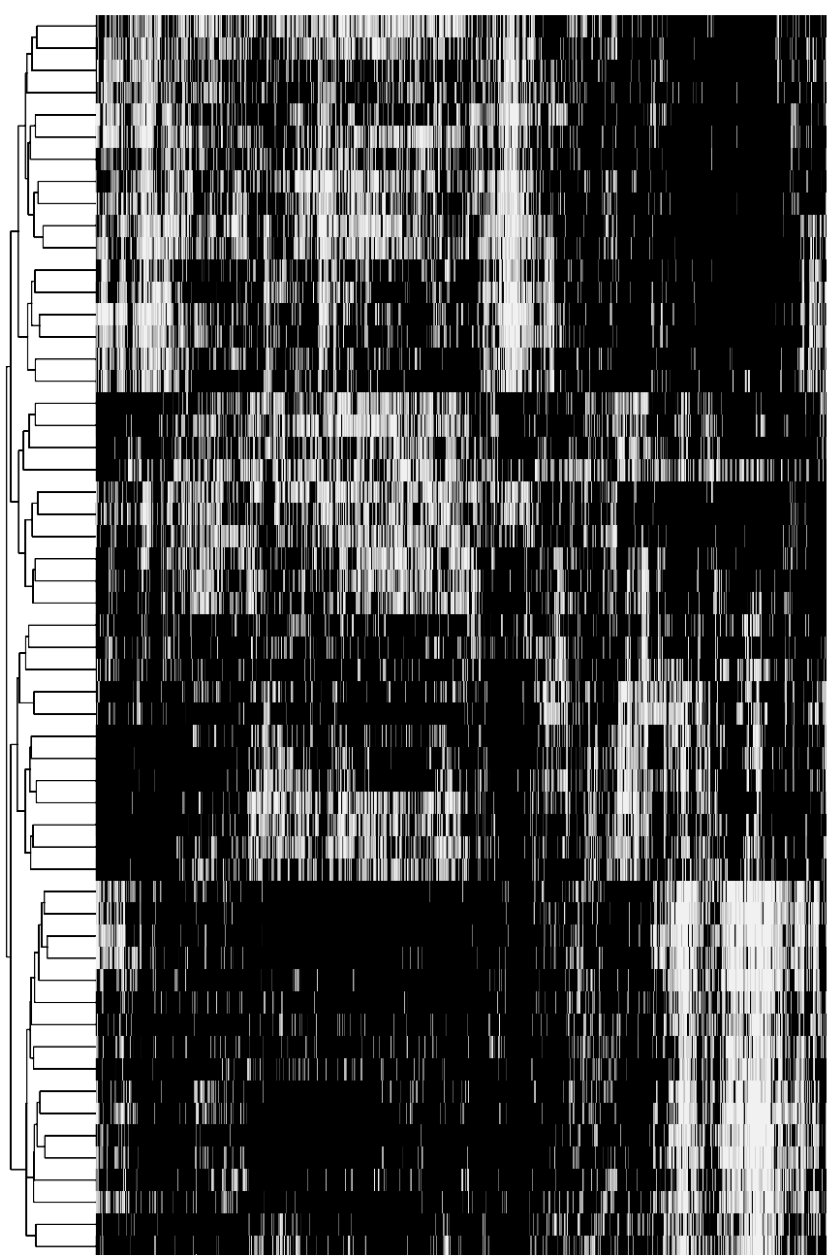

Fig. 2. Data set heat map with cluster configuration identified. The greyscale represents different cluster memebership.

model is an integral part of any statistical analysis and especially fundamental in the Bayesian framework, where prior knowledge is included in the model. A brief model checking analysis is presented below.

1) Model checking: A principled way to evaluate a model is to analyse its out-of-sample predictive performance. The validation process involves comparison of the goodness-of-fit of models with a different number of covariates included. In particular, we analyse the predictive performance of three different models on 1,000 out-of-sample authentication events of LANL data set. Table III shows a comparison of the three following models:

$$
\begin{aligned}
& \text { - Model 1: } \lambda_{i j}(t)=1, \quad\left(\alpha_{1}=0, \alpha_{2}=0\right) \\
& \text { - Model 2: } \lambda_{i j}(t) \propto \exp \left\{\alpha_{1} N_{j}(t)\right\}, \quad\left(\alpha_{2}=0\right)
\end{aligned}
$$


- Model 3: $\lambda_{i j}(t) \propto \exp \left\{\alpha_{1} N_{j}(t)+\alpha_{2 k(i)} N_{k(i) j}(t)\right\}$

The first model represents a null model where the probability of establishing a new connection is the same for each computer. In the second model, cluster-specific effects are not included while the third saturated model includes both overall and cluster-level effects. Furthermore, the analysis was repeated without updating the initial agglomerative clustering configuration jointly with Cox model parameters, in order to evaluate the improvement in the predictive performance of MCMC-based clusters. Comparisons were obtained by using the ratio of the marginal likelihoods for each pair of models. Note that the marginal likelihoods of Model 1 and Model 2 are each invariant to the cluster configuration.

TABLE III

LOG-LIKELIHOOD RATIOS FOR THE DIFFERENT MODELS

\begin{tabular}{c|c|c}
\hline Comparison & LR - $\mathbb{C}$ not updated - & LR - $\mathbb{C}$ updated - \\
\hline \hline Model 3 vs Model 1 & 589.03 & 607.56 \\
Model 3 vs Model 2 & 2.76 & 3.46
\end{tabular}

The results show a significant improvement in the predictive performance of Model 3 with respect to both the null model and Model 2. They also highlight a considerable contribution provided by the cluster-level covariates. Finally, the clusterdegree effect is stronger when cluster configurations are updated jointly with Cox model parameters, confirming an improved predictive performance of the MCMC-based clusters. Finally, a diagnostic tool is used for analysing the convergence of the MCMC algorithm.

2) Convergence diagnosis: A convergence diagnostic assesses the performance of an MCMC sampler by monitoring the convergence of the Markov chain to the stationary distribution. Here a plot of the marginal likelihood for each MCMC iteration is provided in Figure 3 in order to measure the goodness-of-fit of the saturated model. The process appears stationary as the number of iterations increases.

\section{CONCLUSION}

We presented a sequential two-step Bayesian inference procedure aimed at clustering users and modelling new edge formation in the large computer network of Los Alamos National Laboratory. The mechanism of new edge formation was modelled as a Cox proportional hazards model, including both overall and cluster-specific covariates for current computer degrees. Agglomerative model-based clustering was used as a first step of the analysis in order to provide a reliable, initial cluster configuration while an MCMC approach was employed to update the initial cluster configurations jointly with Cox model parameters, with the purpose of improving the predictive performance of the initial clusters.

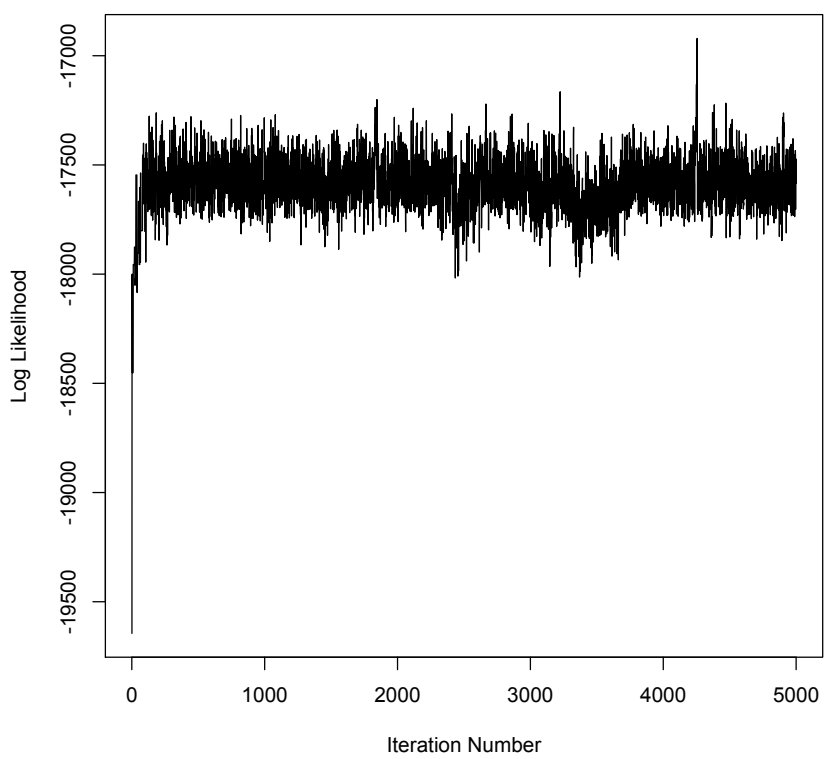

Fig. 3. Marginal likelihood (log) vs. number of MCMC iterations

Results showed a considerable significance of the time-varying computer degree covariates on the predictive probability of observing a particular new connection. Furthermore, the analyses strongly indicate the positive impact of introducing cluster information when modelling the identity of new edges.

\section{REFERENCES}

[1] A. Patcha and J. Park, "An overview of anomaly detection techniques: Existing solutions and latest technological trends," Computer Networks, vol. 51, no. 12, pp. 3448-3470, 2007.

[2] M. H. Cahill, D. Lambert, J. C. Pinheiro, and D. X. Sun, "Detecting fraud in the real world," Computing Reviews, vol. 45, no. 7, p. 447, 2004.

[3] N. Heard and S. Metelli, "Modelling new edge formation in a computer network through bayesian variable selection." IEEE, 2014.

[4] D. R. Cox, "Regression models and life-tables (with discussion)," J. R. Statist. Soc. B, vol. 34, pp. 187-220, 1972.

[5] M. T. S. Kim and M. Vannucci, "Variable selection in clustering via dirichlet process mixture models," Biometrika, vol. 93, pp. 877-893 877-893 877-893, 2006.

[6] R. O. Duda and P. E. Hart, Pattern Classification and Scene Analysis. New Yotk: John Willey \& Sons, 1973.

[7] J. D. Banfield and A. E. Raftery, "Model-based gaussian and nongaussian clustering," Biometrics, vol. 49, pp. 803-821, 1993.

[8] D. J. MacKay, "Bayesian interpolation," Neural Computation, vol. 4, pp. 415-447, 1991.

[9] W. K. Hastings, "Monte carlo sampling methods using markov chains and their applications," Biometrika, vol. 57, no. 1, pp. 97-109, 1970.

[10] A. D. Kent, "User-computer authentication associations in time," Los Alamos National Laboratory, 2014. 